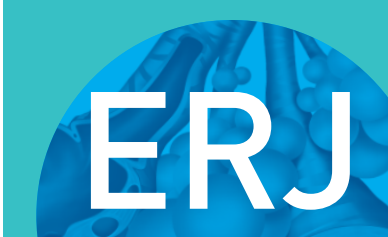

open research

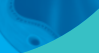

\title{
Influence of age, sex and hospitalisation on the administration of tuberculosis medication: an evaluation of routine data from a German health insurer
}

\author{
Jan F. Kersten ${ }^{1}$, Stefanie Wobbe-Ribinski ${ }^{2}$, Roland Diel ${ }^{3,4}$, Albert Nienhaus $^{1,3}$ \\ and Anja Schablon ${ }^{1}$
}

Affiliations: ${ }^{1}$ Competence Center for Epidemiology and Health Services Research for Healthcare Professionals (CVcare), Institute for Health Services Research in Dermatology and Nursing (IVDP), University Medical Center Hamburg-Eppendorf (UKE), Hamburg, Germany. ${ }^{2}$ DAK-Gesundheit, Hamburg, Germany. ${ }^{3}$ Dept of Occupational Medicine, Public Health and Hazardous Substances, Institution for Statutory Accident Insurance and Prevention in the Health and Welfare Services (BGW), Hamburg, Germany. ${ }^{4}$ Institute for Epidemiology, University Hospital Schleswig-Holstein, Campus Kiel, Kiel, Germany.

Correspondence: Jan F. Kersten, Martinistrasse 52, 20246 Hamburg, Germany. E-mail: j.kerstenđuke.de

\section{ABSTRACT}

Background: So far, there is no reliable information on the drugs actually taken by tuberculosis patients. With billing data from a large German health insurance company, valid data from practice will be used for analysis. The objective here is to use the claims data of a health insurer to gain an insight into the prescriptions issued to patients with tuberculosis in Germany.

Methods: The study design encompasses a longitudinal, analytical observational study of selected insurance holders. Descriptive analyses of the outpatient drug supply of pulmonary tuberculosis patients are determined for 6 payroll years. We have studied whether different doses of tuberculosis medication are associated with age, sex, inpatient status and comorbidity. Quantile regression is used as a method to identify subgroups or characteristic dosages.

Results: The number of defined daily doses prescribed per patient varies among insurance holders and encompasses widely differing timeframes. Higher doses are observed with increasing age, as well as in patients with tuberculosis-related hospitalisations. The sex of the patient has no identifiable effect on the prescribed doses for any of the first-line tuberculosis drugs. Comorbidity partially has a significant impact on the duration and intensity of tuberculosis drug prescriptions.

$@$ @RSpublications

For tuberculosis patients, the doses of the prescribed four first-line drugs are higher for patients having received a main diagnosis of tuberculosis upon hospital discharge than for outpatients without prior hospitalisation https://bit.ly/3dFw3Qq

Cite this article as: Kersten JF, Wobbe-Ribinski S, Diel R, et al. Influence of age, sex and hospitalisation on the administration of tuberculosis medication: an evaluation of routine data from a German health insurer. ERJ Open Res 2020; 6: 00369-2019 [https://doi.org/10.1183/23120541.003692019].

This article has supplementary material available from openres.ersjournals.com

Received: 21 Jan 2020 | Accepted after revision: 6 May 2020

Copyright $\odot$ ERS 2020. This article is open access and distributed under the terms of the Creative Commons Attribution Non-Commercial Licence 4.0. 


\section{Introduction}

Every $3 \mathrm{~s}$, a person is infected with Mycobacterium tuberculosis and 5-10\% of the immunocompetent carriers develop active tuberculosis (TB) over the course of their lives [1]. As far as active TB is concerned, a number of Western European countries have been considered to be low-incidence countries for several years now. However, TB is still the infectious disease with the highest number of deaths worldwide [2-4].

The conventional method of treating TB consists of the administration of multiple drugs, known as anti-TB drugs, for a period of 6 months. In the initial phase, a combination of four drugs is taken to prevent resistance and quickly reduce the number of reproductive pathogens. During this initial 2-month phase, a combination of the drugs rifampicin (RMP), isoniazid (INH), pyrazinamide (PZA) and ethambutol (EMB) is given. Streptomycin (SM) may also be prescribed as an alternative to EMB, although this is rarely the case nowadays and is mainly used for cases of TB affecting the central nervous system and, if there is a proven susceptibility, for multidrug-resistant strains. The standard "short course" therapy with proven sensitivity to all four of these first-line drugs involves treatment with a combination of RMP and INH for another 4 months. The required duration of the drug treatment may far exceed the conventional 6-month period; multidrug-resistant strains may necessitate treatment periods of 20 months, even under ideal conditions $[5,6]$.

The World Health Organization (WHO) adopted a strategy in 2014 that aims to drastically reduce the spread of TB by 2035. Compliance with therapy standards is essential to achieve the goals of this End TB Strategy $[7,8]$. In line with WHO guidelines [9], consensus-based S2 standards for the treatment of TB in adults and in children $[6,10]$ were developed in Germany to specify the ideal doses and treatment durations for both the conventional form of TB and for special drug-resistant forms and specific patient groups [11].

\section{Aims of the study}

The data of a health insurer are being used here to give an insight into how drugs are prescribed to patients with active TB. This will enable to analyse factors that might influence TB treatment. In addition to age, sex and medical risk factors for $\mathrm{TB}$, the influence of the form of treatment (inpatient combined with outpatient care or outpatient care only) on the prescribed doses of drugs will be analysed.

\section{Materials and methods \\ Study design}

The study design encompasses a longitudinal, analytical observational study of selected insurance holders of the DAK-Gesundheit, Germany's third-largest statutory health insurance fund.

The analysis is based on personal data of insurance holders at DAK-Gesundheit. These data represent a complete collection of data for insurance holders in the period from 1 January, 2009 to 31 December, 2014 Insurance holders could appear in the database by one of two means: 1) They were diagnosed with TB. The diagnoses in this case are those recorded in the claims data of DAK-Gesundheit. For outpatients, confirmed diagnoses in the course of outpatient consultations are required, and only main diagnoses recorded upon discharge are recognised for inpatients. 2) Insurance holders collected prescriptions with drugs for the treatment of TB. At least one prescription for a TB-specific drug must have been redeemed in the period under consideration (2009-2014). Information on the person (i.e. year of birth and sex) was required. Moreover, only the billed drugs with a correct centrally administered pharmaceutical identification number ('Pharmazentralnummer' (PZN)), were used. The coding in the numerical sequence of the PZN ensures a clear identification of a drug, its purpose, formulation and package size, supporting the elimination of unsuitable drug usage. The selection criteria produced 1817 insurance holders (figure 1).

\section{Ethical statement}

No decision of the Ethics Committee is required for this investigation of claims data. In accordance with the Professional Code for Physicians in Hamburg (Art. 15, 1, Status of 10.03.2014) and the Chamber Legislation for Medical Professions in the Federal State of Hamburg it is only necessary to obtain advice on questions of professional ethics and professional conduct from an ethics committee if data which can be traced to a particular individual are being used in a research project.

\section{Target variables}

An analysis and count of 14 anti-TB drugs listed in the prescriptions of the anatomical therapeutic chemical (ATC) classification system was performed. The outpatient prescriptions of the four current first-line drugs were studied using the innovative quantile regression method [12-14]. In our case, the use of quantile regression studied the effect of independent variables on various levels of intensity of the drugs. This enables a study of how each of the factors of influence differ in their impact as drug intensity is varied (i.e. variations in the dose of the drug studied as the reference measure) [15]. 


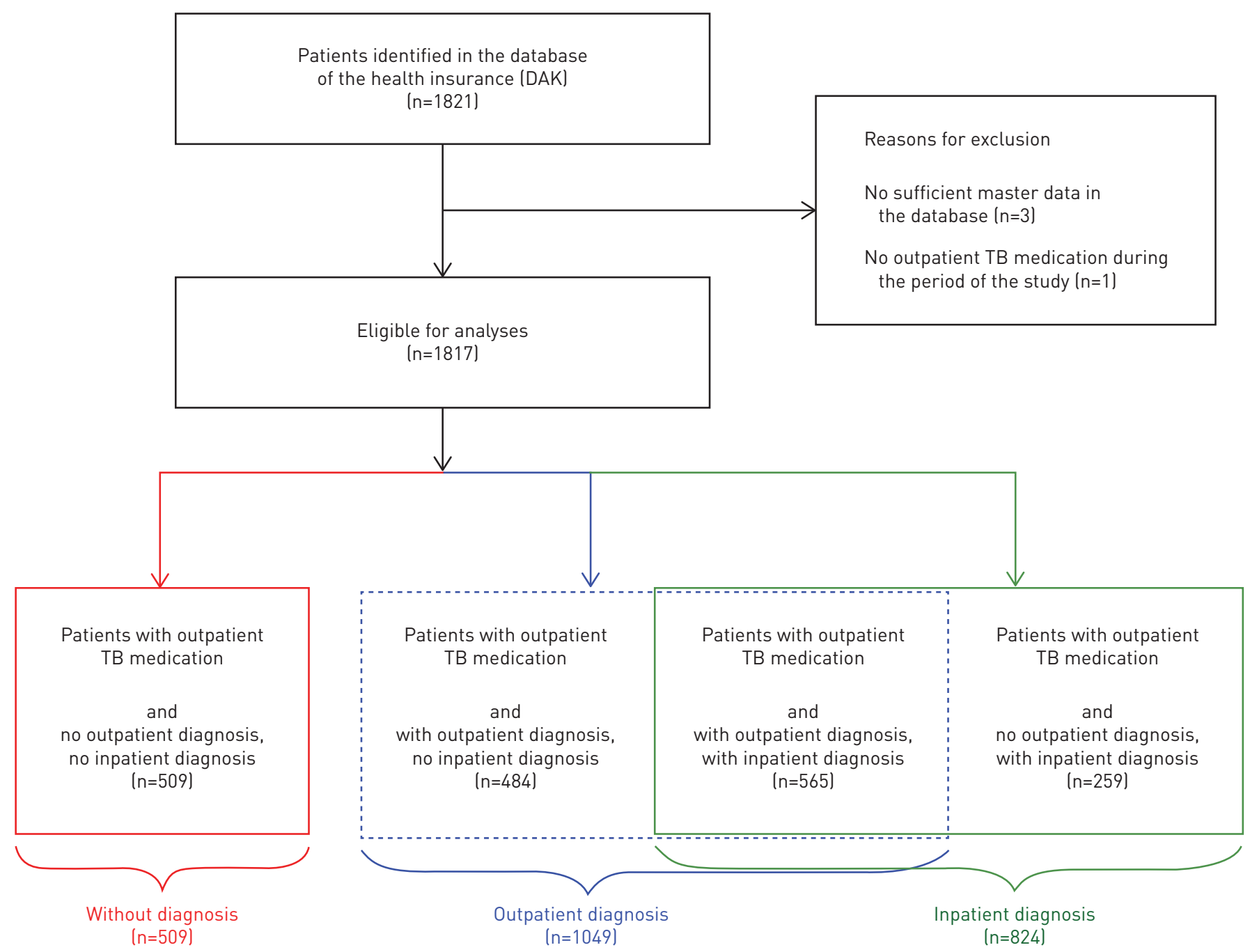

FIGURE 1 Composition of the study population (n=1817). TB: tuberculosis.

Temporary discharges from hospitals, something that is not uncommon in practice due to patient wishes or organisational needs, were recognised as a single hospital admission for the purpose of drug administration if the period until discharge period did not last more than 3 days. Patients treated for TB in a hospital were assumed to have received drug treatment from the fourth day onwards in relation to the period of drug administration [16].

All personal health-related data in this sample was collected and processed [17] following the European General Data Protection Regulation and the German Social Code Book X [18]. The necessary approval was granted by the competent regulatory authority, contractual agreements were concluded on the collection and use of the data [19]. Internal data protection rules were applied for the pseudonymisation of data collected by DAK-Gesundheit, including the personally identifiable health insurance account numbers and identifying information of service providers [20].

A one-time snapshot extract of the treatment database of DAK-Gesundheit was prepared and submitted for the period from 1 January, 2009 to 31 December, 2014.

\section{Definitions}

TB of the respiratory tract is defined as diagnosed in accordance with the ICD-10 GM (German Modification) codes and comprises the three-digit codes A15 "Respiratory TB (confirmed by bacteriological, molecular biological or histological means)" and A16 "Respiratory TB (not confirmed by bacteriological, molecular biological or histological means)". The ICD-10-GM three-digit code A19 refers to miliary TB [21]. The ICD-10 three-digit codes A15 and A16 will also be referred to jointly as "pulmonary $\mathrm{TB}$ " in the following. 
Variables and information sources

These data were sourced from the routine data of DAK-Gesundheit and comprises pseudonymised master data (year of birth, sex, start of insurance, end of insurance), outpatient data (confirmed diagnoses), outpatient drug prescriptions (ATC codes and PZN) with corresponding dispensing dates and hospital data (date of admission, date of discharge, diagnosis and diagnostic status).

This study considers patients with pulmonary and/or miliary TB. The current first-line drugs (RMP, INH, PZA and EMB) as well as second-line drugs (streptomycin, rifabutin, moxifloxacin, levofloxacin, protionamide, amikacin, aminosalicylic acid, delamanid, terizidone and bedaquiline) were considered here as TB-specific drugs. The latter drugs are used mostly in patients who exhibit intolerances or resistances to drugs, where the first-line drugs are unable to have their intended effect.

Due to statutory data retention requirements, the data encompass different time periods (for the inpatient diagnoses, data are available on insurance holders for the entire period from 2009 to 2014). These data enable an analysis of hospitalisation duration and discharge diagnoses. For the outpatient diagnoses, the obtainable time period is limited, as German health insurers are required by law to delete these diagnostic data after 4 years [22]. Due to this legal restriction, outpatient diagnoses are only available for the years from 2012 to 2014. There is also a specific factor to take into consideration regarding drugs administered during hospital stays. Data on specific drugs during the hospital stays are not transmitted to the health insurers, which is why the drugs administered in the hospital are not included in the analyses presented below. The database relating to specific drugs is therefore limited to outpatient prescriptions.

The prescription rates for active ingredient groups (first based on the ATC classification) are presented here. Based on the additional information for the PZN of the billed drug, the number of the received defined daily doses (DDDs) for the active ingredients is determined [23, 24]. This enables the information on the ATC classification to be supplemented with the package size recorded under the PZN, which indicates the number of standardised DDDs of the included active ingredients. The DDDs and the time period between the prescription redemptions allow a measure to be defined that represents the theoretical compliance. The number of DDDs identified using the billed prescription only allows for an estimate of the drugs taken, because neither the specific prescription by the physician nor the correct use of the drugs by the patient have been documented.

For the purposes of statistical inference, these DDDs related to the period in which each insurance holder had the characteristic TB drugs available for use. Accordingly, the usage time starts upon the first prescription issued and ends upon the last prescription used for characteristic TB drugs. All drugs with the exception of the last prescription are considered in terms of the DDD available per day for the related analyses. Both measures, the DDD and the derivative quotient, primarily serve as a means of estimating therapy compliance and do not need to be consistent with the recommended drugs nor with the actual prescribed doses [24]. The DDD for the four first-line drugs for standard treatments were $600 \mathrm{mg}$ for RMP, $300 \mathrm{mg}$ for INH, $1750 \mathrm{mg}$ for PZA and $1200 \mathrm{mg}$ for EMB in the period under study.

Miliary TB is initially treated in exactly the same way as a complication-free case of pulmonary TB. If response to therapy is poor (e.g. due to immunosuppression), special second-line drugs are used. The drug treatment of miliary TB is therefore addressed separately (table S1). The impact of known risk factors on the duration of treatment and the use of drugs has been studied in terms of differences relative to the remaining population. Risk factors include diabetes mellitus, HIV, renal failure, alcoholism [6], Crohn's disease and antibiotic resistance. These risk factors have been identified from the confirmed outpatient diagnoses (categorised in accordance with the ICD) and from the hospital discharge diagnoses. Differences in age and sex were also identified in terms of drug treatment durations and hospital stay durations.

\section{Statistical analysis}

Counts and categorical variables are shown as frequencies and proportions (as a percentage). Continuous variables are reported both as arithmetic mean values with SD and as median values with range. Group comparisons are performed using a t-test or Fisher's exact test based on the scale of measure of each variable, as appropriate. If necessary, the continuous scales are log-converted beforehand to satisfy the distribution requirements. The values shown in the text have been transformed back to their original scale. To study the derived individual compliance (total DDDs per total days) in relation to age, sex and hospital discharge, quantile regressions were performed for the four first-line drugs (RMP, INH, PZA, EMB) on the adult insurance holders at the time of the initial diagnosis of pulmonary $\mathrm{TB}$ using the interior algorithm with $95 \%$ confidence interval.

A relatively new analysis method, quantile regression, was chosen. The objective of this method is to identify subgroups and any potential inconsistent effects in terms of how TB drugs are supplied. The 
advantage of quantile regression over generalised linear models is that no distribution requirements need to be met.

Quantile regression examines the entire spectrum of the available medication intensity, whether an influence of the investigated, independent variables on adherence can be statistically demonstrated. This method of compliance analysis examines insurance holders who have redeemed at least two prescriptions for TB drugs in the study period. The compliance quotients for first-line drugs (RMP, INH, PZA, EMB) were studied using quantile regression in terms of the impact of the variables age, sex and main diagnosis upon discharge from hospital. The effect of the three factors of influence is stated for the four compliance quotients. All analyses were prepared using the statistical analysis software R 3.5.2 [25] and SAS 9.4 [26].

Patient and public involvement

This was a secondary data analysis and therefore there was no direct patient or public involvement.

\section{Results}

\section{Selection of study population}

Of the 1817 patients under study, there were exactly 206 insurance holders for the year 2009 who first appeared in the database as a result of a TB drug prescription. Regarding the additional insurance holders, the diagnosis figures for pulmonary TB is in a range of 171 to 262 patients, while the diagnosis figures for miliary TB are between 4 and 10 patients each year (figure 2). In addition to 32 cases of miliary TB diagnosed in hospitals, there were 18 outpatient diagnoses of miliary TB.

\section{Descriptive results}

The share of women was $49 \%$ and the average age upon the initial TB diagnosis or prescription of a TB-specific drug was 51 years. The proportion of older insurance holders ( $>64$ years of age) was 35\%, whereas the average age was 51 years among men ( \pm 24 years) and 52 years among women ( \pm 23 years). Table 1 provides an overview of the descriptive variables for the population.

Over the 6 years there were 1172 main diagnoses upon discharge from hospital for 824 insurance holders, as multiple hospital stays resulted in individual insurance holders being referenced several times. This meant that there were 567 insurance holders with one hospital discharge, 201 with two, 34 with three, 14 with four, 5 with five, 2 with six and 1 insurance holder with eight main discharge diagnoses of TB within the hospital discharge period from 2009 to 2014. The number of prescribed daily doses is shown for the active ingredients over the years in table 2. Of the 14 studied drugs, 13 were prescribed in the period under study. For 905 patients, the simultaneous administration of three or four different drugs is documented, which is conventional for initial medication. Of these patients, $722(79.8 \%)$ received therapy comprising the four first-line drugs RMP, INH, PZA and EMB, while treatment comprising the three drugs RMP, INH and PZA was administered to 183 patients $(20.2 \%)$.

Overall, 260 patients (14.3\%) had a derived medication period of over 6 months from the first to the last TB drug prescription. 658 (36.2\%) of insurance holders received TB treatment lasting more than 2 months in the period under study.

For patients exhibiting more than one redeemed prescription in the database, the average DDDs per day are shown in table 3. It should be noted here that only the drugs INH and RMP are usually taken

FIGURE 2 Year of the first diagnosis or first medication with specific anti-tuberculosis (TB) drugs ( $\mathrm{n}=1817)$.

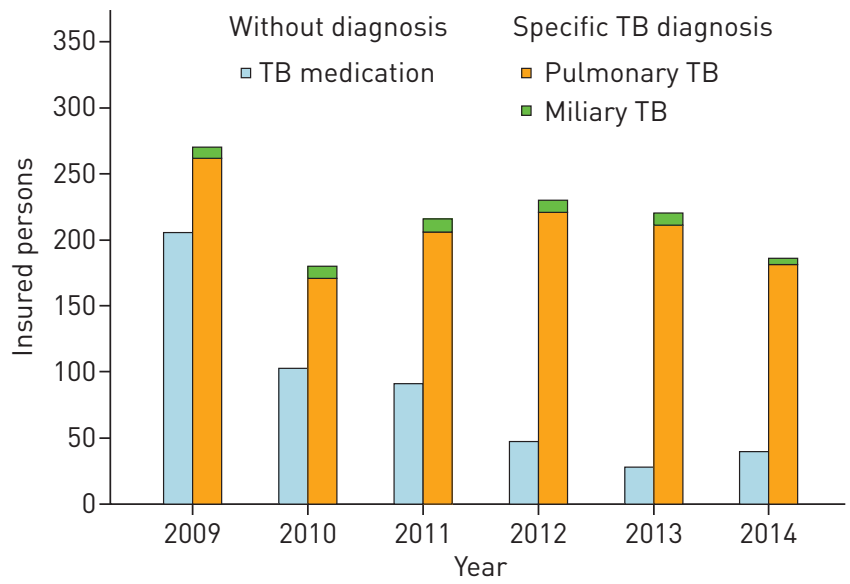




\begin{tabular}{|c|c|c|c|}
\hline Variable & $\begin{array}{c}\text { Women } \\
\text { n (row } \\
\text { percentage) }\end{array}$ & $\begin{array}{c}\text { Men } \\
\text { n (row } \\
\text { percentage) }\end{array}$ & $\begin{array}{c}\text { Overall } \\
\text { n (column } \\
\text { percentage) }\end{array}$ \\
\hline \multicolumn{4}{|l|}{ Age ${ }^{\#}$ years } \\
\hline Mean $\pm S D$ & $51.8 \pm 23.3$ & $51.0 \pm 23.7$ & $51.4 \pm 23.5$ \\
\hline Median (range) & $56(0-93)$ & $54(0-92)$ & $55(0-93)$ \\
\hline \multicolumn{4}{|l|}{ ICD-10 diagnosis (3-digit) } \\
\hline A15 (confirmed respiratory TB) & $263(46.6 \%)$ & $301(53.4 \%)$ & $564(31.4 \%)$ \\
\hline A16 (unconfirmed respiratory TB) & $439(48.8 \%)$ & $461(51.2 \%)$ & $900(49.5 \%)$ \\
\hline A19 (miliary TB) & $27(54.0 \%)$ & $23(46.0 \%)$ & $50(2.8 \%)$ \\
\hline \multicolumn{4}{|l|}{ Death, unknown cause } \\
\hline Number & $105(35.1 \%)$ & $194(64.9 \%)$ & $299(16.5 \%)$ \\
\hline \multicolumn{4}{|l|}{ Age years } \\
\hline Mean \pm SD & $74.1 \pm 11.7$ & $72.8 \pm 11.7$ & $73.2 \pm 11.7$ \\
\hline Median (range) & 76 (35-93) & $74(23-92)$ & 75 (23-93) \\
\hline \multicolumn{4}{|c|}{ Work incapacity certificate (reason: A15, A16, A19) } \\
\hline Number of cases of work incapacity & $58(45.3 \%)$ & $70(54.7 \%)$ & $128(7.0 \%)$ \\
\hline \multicolumn{4}{|l|}{ Work incapacity period days } \\
\hline Mean $\pm S D$ & $141.0 \pm 137.4$ & $133.0 \pm 134.9$ & $136.6 \pm 135.5$ \\
\hline Median (range) & $113.5(1-550)$ & $98(2-547)$ & $99.5(1-550)$ \\
\hline $\begin{array}{l}\text { Number of cases of work incapacity involving } \\
\text { disease of the respiratory system }{ }^{+}\end{array}$ & $167(49.1 \%)$ & $173(50.9 \%)$ & $340(18.7 \%)$ \\
\hline TB as main diagnosis upon hospital discharge & $344(41.7 \%)$ & $480(58.3 \%)$ & $824(45.3 \%)$ \\
\hline TB as outpatient diagnosis & $287(47.7 \%)$ & $315(52.3 \%)$ & $602(33.1 \%)$ \\
\hline
\end{tabular}

throughout the entire treatment period, meaning that only these median values are observed at a value of above one.

Table 4 shows the figures for documented comorbidity in the period from 2009 to 2014 . The number of comorbidity cases is shown both in aggregated form and stratified by outpatients and inpatients being admitted due to TB. The most common comorbidity among the insurance holders in the study was with diabetes mellitus, renal failure and alcoholism. Table 5 shows the difference in the length of the hospital

TABLE 2 Number of outpatient prescription-defined daily doses for tuberculosis drugs for the years 2009 to $2014(n=1817)$

\begin{tabular}{|c|c|c|c|c|c|c|c|}
\hline & \multirow{2}{*}{$\begin{array}{l}\text { Active ingredient (number of } \\
\text { patients) }\end{array}$} & \multicolumn{6}{|c|}{ Year } \\
\hline & & 2009 & 2010 & 2011 & 2012 & 2013 & 2014 \\
\hline \multirow[t]{4}{*}{ First-line drugs } & Isoniazid (n=1563) & 64358 & 50100 & 52400 & 52375 & 45383 & 45025 \\
\hline & Rifampicin ( $n=1458$ ) & 54860 & 46979 & 47921 & 43131 & 37340 & 39152 \\
\hline & Ethambutol $(n=1110)$ & 23498 & 22650 & 24687 & 21783 & 18638 & 17438 \\
\hline & Pyrazinamide ( $n=991)$ & 15127 & 12713 & 11007 & 11650 & 10533 & 10850 \\
\hline \multirow{10}{*}{$\begin{array}{l}\text { Second-line } \\
\text { drugs }\end{array}$} & Protionamide $(n=18)$ & 333.3 & 666.7 & 616.7 & 183.3 & 183.3 & 116.7 \\
\hline & Amikacin $(n=9)$ & 0 & 10 & 47.5 & 0 & 5 & 70 \\
\hline & Aminosalicylic acid ( $n=1)$ & 0 & 0 & 0 & 0 & 0 & 19.3 \\
\hline & Delamanid $(n=1)$ & 0 & 0 & 0 & 0 & 0 & 10 \\
\hline & Rifabutin ( $n=96$ ) & 3960 & 5190 & 6420 & 6030 & 6180 & 4860 \\
\hline & Terizidone $(n=5)$ & 14.3 & 28.6 & 0 & 157.1 & 71.4 & 0 \\
\hline & Streptomycin $(n=14)$ & 160 & 50 & 20 & 100 & 0 & 0 \\
\hline & Bedaquiline $(n=0)$ & 0 & 0 & 0 & 0 & 0 & 0 \\
\hline & Moxifloxacin ( $n=304$ ) & 779 & 1376 & 2003 & 1578 & 1698 & 1245 \\
\hline & Levofloxacin ( $n=363$ ) & 938.5 & 1239 & 1145 & 1133.5 & 1049.5 & 768.5 \\
\hline
\end{tabular}


TABLE 3 Defined daily doses per medication day (difference: last prescription, first prescription with $n=727$ insurance holders having at least two redeemed prescriptions for tuberculosis drugs under study)

\begin{tabular}{|c|c|c|c|c|c|c|c|}
\hline & Active ingredient (patients) & Minimum & 1st quartile & Median & Mean & 3rd quartile & Maximum \\
\hline \multirow{2}{*}{ First-line drugs } & Rifampicin $(n=641)$ & 0.026 & 0.791 & 1.359 & 2.380 & 2.198 & 130.000 \\
\hline & Pyrazinamide $(n=527)$ & 0.018 & 0.293 & 0.514 & 1.007 & 0.851 & 66.666 \\
\hline \multirow[t]{8}{*}{ Second-line drugs } & Protionamide $(n=7)$ & 0.017 & 0.082 & 0.441 & 1.104 & 0.989 & 5.128 \\
\hline & Amikacin $(n=4)$ & 0.033 & 0.104 & 0.130 & 0.362 & 0.387 & 1.154 \\
\hline & Rifabutin ( $n=25)$ & 0.047 & 0.526 & 0.737 & 1.448 & 1.826 & 7.826 \\
\hline & Terizidone $(n=4)$ & 0.044 & 0.070 & 0.144 & 0.174 & 0.248 & 0.365 \\
\hline & Streptomycin $(n=10)$ & 0.010 & 0.054 & 0.082 & 1.952 & 0.148 & 20.000 \\
\hline & Bedaquiline $(n=0)$ & 0.000 & 0.000 & 0.000 & 0.000 & 0.000 & 0.000 \\
\hline & Moxifloxacin (n=144) & 0.004 & 0.040 & 0.098 & 0.352 & 0.228 & 7.500 \\
\hline & Levofloxacin ( $n=165$ ) & 0.003 & 0.041 & 0.082 & 0.179 & 0.161 & 2.857 \\
\hline
\end{tabular}

stay and length in drug treatment compared to those insurance holders without the relevant comorbidity. The differences are additional days observed in patients with the diagnosed comorbidity compared to the rest of the population. It has been demonstrated with statistical significance that patients with at least one comorbidity spend a longer period during a hospital stay of an average of 27.2 days, especially where there is comorbidity with alcoholism (48 days) and antibiotic resistance (63 days). HIV caused a significant increase in TB drug administration (112 days).

Patients of advanced age ( $>64$ years) trended towards longer hospital stays and lower drug treatment durations, although the effects were not statistically significant. Male patients exhibited on average a shorter TB drug treatment duration compared to women. Patients with a documented TB diagnosis exhibited higher values for hospital stay durations and drug treatment durations on average. The effects of the diagnosis were (with the exception of the hospital stay duration amongst pulmonary TB patients) all statistically significant. Insurance holders with documented miliary TB exhibited hospitalisation of 35 days longer and a drug treatment duration of 117 days longer compared to the rest of the population (table 6).

\section{Quantile regression}

The following compliance analyses examine all 727 adult insurance holders who have received at least two prescriptions for TB drugs in the study period. The results for the four first-line drugs are described below.

\section{TABLE 4 Comorbidity among insurance holders (multiple assignments possible)}

\begin{tabular}{|c|c|c|c|}
\hline Comorbidity & $\begin{array}{l}\text { No hospital stay due to } \\
\text { TB n (row percentage) }\end{array}$ & $\begin{array}{l}\text { Hospital stay due to TB } \\
\text { n (row percentage) }\end{array}$ & $\begin{array}{l}\text { Number (proportion of } \\
\text { total population) }\end{array}$ \\
\hline Diabetes mellitus & $105(30.9 \%)$ & $235(69.1 \%)$ & $340(18.7 \%)$ \\
\hline HIV & $9(23.1 \%)$ & $30(76.9 \%)$ & $39(2.1 \%)$ \\
\hline Renal failure & $52(16.9 \%)$ & $256(83.1 \%)$ & $308(17.0 \%)$ \\
\hline Alcoholism & $54(28.7 \%)$ & 134 (71.3\%) & $188(10.3 \%)$ \\
\hline Crohn's disease & $15(39.5 \%)$ & $23(60.5 \%)$ & $38(2.1 \%)$ \\
\hline Antibiotic resistance & $7(17.9 \%)$ & 32 (82.1\%) & $39(2.1 \%)$ \\
\hline $\begin{array}{l}\text { Patients with at least } \\
\text { one comorbidity" }\end{array}$ & $168(22.9 \%)$ & $565(77.1 \%)$ & $733(40.3 \%)$ \\
\hline $\begin{array}{l}\text { Patients with no known } \\
\text { comorbidity }\end{array}$ & $825(76.1 \%)$ & $259(23.9 \%)$ & $1084(59.7 \%)$ \\
\hline
\end{tabular}




\begin{tabular}{|c|c|c|}
\hline \multirow[t]{2}{*}{ Comorbidity \# } & \multicolumn{2}{|c|}{ Comorbidity effect difference days } \\
\hline & $\begin{array}{l}\text { Additional hospital stay duration of } \\
\text { hospitalised patients }(n=824)\end{array}$ & $\begin{array}{l}\text { Additional drug treatment } \\
\text { duration }(n=1817)\end{array}$ \\
\hline Diabetes mellitus & $-0.3(p=0.981)$ & $4.6(p=0.766)$ \\
\hline HIV & $25.3(p=0.399)$ & $111.9(p=0.007)^{* *}$ \\
\hline Renal failure & $-3.7(p=0.761)$ & $14.8(p=0.353)$ \\
\hline Alcoholism & $48.0(p<0.001)^{* * *}$ & $14.6(p=0.456)$ \\
\hline Crohn's disease & $-32.2(p=0.402)$ & $77.1(p=0.065)$ \\
\hline Antibiotic resistance & $63.2(p=0.016)^{*}$ & $70.1(p=0.093)$ \\
\hline $\begin{array}{l}\text { Patients with at least one } \\
\text { comorbidity }\end{array}$ & $27.2(p=0.003)^{* *}$ & $25.0(p=0.040)^{*}$ \\
\hline
\end{tabular}

\section{Rifampicin}

There is no demonstrable effect of age or sex on compliance.

Another factor that we presumed to affect drug administration was hospital discharge. Our data show that the effect of a hospital discharge, with the exception of very low doses, is around one DDD higher. It is almost entirely independent on the initial dose. Insurance holders exhibiting a very high RMP dose even had their hospital discharge associated with an increase in the dose of more than two DDDs (figure 3).

\section{Isoniazid}

There is no observable effect of age with isoniazid therapy. Hospital discharge is associated with a statistically significantly higher compliance, which seems to remain practically constant at around 0.9 . Sex has no effect on INH therapy in the lower compliance range, although the DDDs are around 0.4 higher for men than for women in the higher compliance range (figure 4).

\section{Ethambutol}

There is no demonstrable effect of sex, although compliance is higher among male patients with higher doses. Age has no demonstrable significant effect on EMB compliance. The hospitalisation effect with this therapy differs significantly from zero and is well above 0.5 units, especially where compliance is higher (figure S1).

\section{Pyrazinamide}

Age and sex are not linked with PZA compliance, while hospital discharges are linked to an approximately 0.5 DDD increase in PZA adherence in the medium and higher compliance range (figure S2).

TABLE 6 Hospital stay durations and drug treatment durations in relation to sociodemographic factors and diagnoses, each compared to the rest of the population

Variable

Effect difference days

Additional hospital stay duration of hospitalised patients ( $\mathrm{n}=824)$

Age $>64$ years

Sex male

Diagnosed

pulmonary TB

Diagnosed miliary

TB $4.4(p=0.603)$

$1.5(p=0.855)$

$7.0(p=0.411)$

$34.9(p=0.001) * *$
Additional drug treatment duration ( $n=1817)$

$-5.1(p=0.479)$

$-21.6(p=0.002) * *$

$42.5(p<0.001)^{* * *}$

$117.4(p=0.009) * *$

TB: tuberculosis. ${ }^{* *}: \mathrm{p}<0.01 ;{ }^{* * *}: \mathrm{p}<0.001$ 

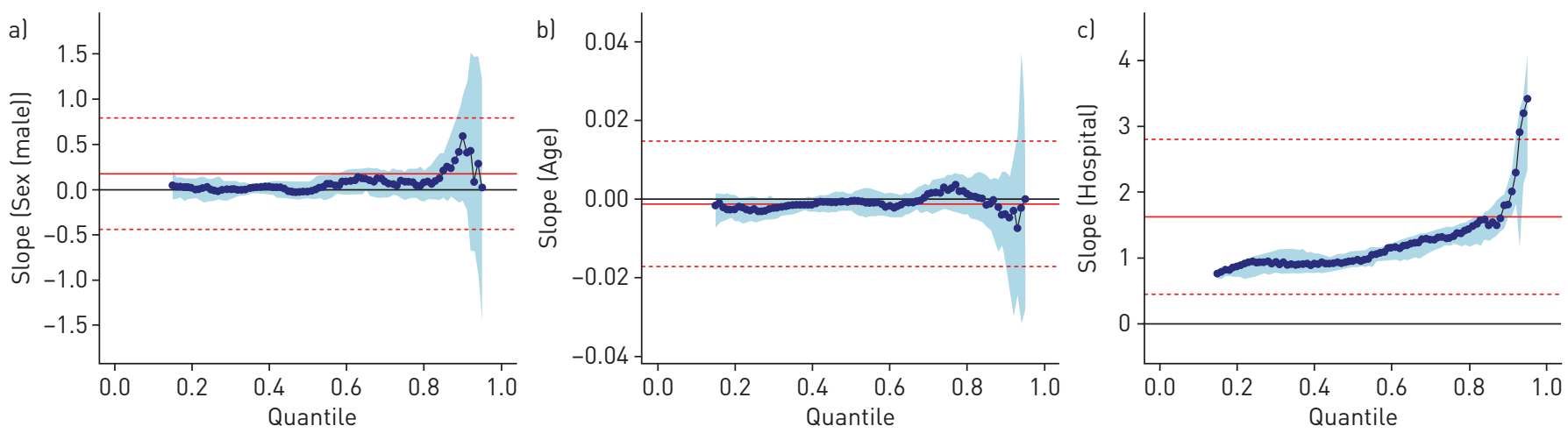

FIGURE 3 Quantile regression for the reference measure rifampicin (defined daily doses per day) on the variables (a) sex, (b) age and (c) hospital discharge $(n=727)$. Blue points represent the estimators for the particular quantile of the drug dose that was available per day. The light blue areas highlight the corresponding $95 \%$ confidence intervals. Useful for comparison is the solid red line representing the constant estimation of a standard regression model.

\section{Summary of results}

The analyses have shown that insurance holders being diagnosed with TB upon discharge from hospital receive a statistically significantly higher number of prescriptions than those insurance holders diagnosed solely on an outpatient basis. It is not possible to determine on the basis of the data used whether this difference is attributable to a different approach to how prescriptions are issued or to differences in morbidity between patients initially admitted to hospital for treatment and patients exclusively treated as outpatients. More extensive studies are required here.

Over one-third of insurance holders received TB treatment lasting more than 2 months in the period under study. The rate of insurance holders receiving work incapacity certificates in the study period due to TB was $7 \%$.

The recommendation issued until 2011 by the German Central Committee against TB (namely to use just three drugs as an initial therapy in mild cases) was found to have been followed in 183 cases. The recommendation was subsequently expanded to include EMB, which is reflected in the increasing prescription rates of this active ingredient $[6,11]$. However, both recommendations were suitable here, as no relevant changes to dose regimens resulted from the changed recommendations.

\section{Discussion}

To our knowledge, this is the first longitudinal study of routine data from a German health insurer on the effect of age, sex and hospitalisation on the administration of TB drugs to patients.

The analysis of the four first-line drugs has shown that patients having received a main diagnosis of TB upon hospital discharge would redeem prescriptions that mandated higher doses of TB drugs. There was no demonstrable age curve, even if the intensity of the prescriptions did tend to rise with increasing age.
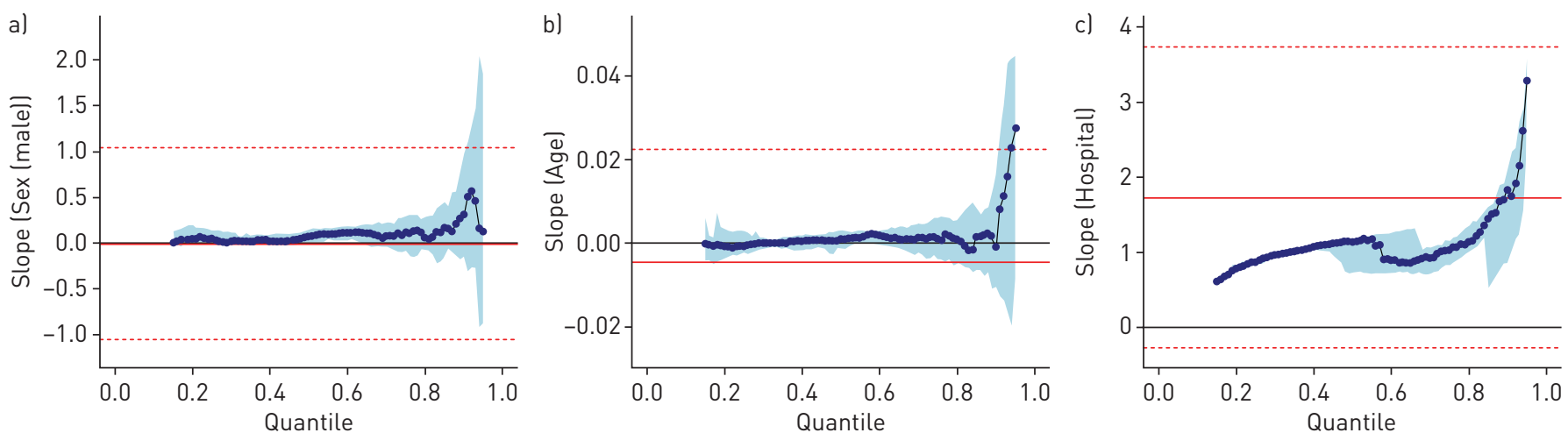

FIGURE 4 Quantile regression for the reference measure isoniazid (defined daily doses per day) on the variables (a) sex, (b) age and (c) hospital discharge ( $n=727)$. Blue points represent the estimators for the particular quantile of the drug dose that was available per day. The light blue areas highlight the corresponding $95 \%$ confidence intervals. Useful for comparison is the solid red line representing the constant estimation of a standard regression model. 
Our analyses have shown with all four therapy compliance levels that firstly, age is not associated with higher compliance levels, secondly, insurance holders having a TB diagnosis upon discharge from hospital exhibit higher compliance levels, and thirdly, male insurance holders are linked to a somewhat higher compliance. However, the latter case is not statistically significant for any of the first-line drugs.

Comorbidity with alcoholism and antibiotic resistance resulted in longer hospital stays. A longer drug treatment duration was demonstrated in patients infected with HIV.

A relatively large number of insurance holders, consisting of TB patients, were included in the analysis. One possible explanation for the identified, sometimes very short prescription times is that patients initially treated with $\mathrm{TB}$ drugs were subsequently found to have received a misdiagnosis. This specific health insurer exhibits a number of particularities in terms of the structure of the insurance holder group under study that should not go unmentioned here; it is a health insurance fund that has evolved from the oldest health insurer in Germany with a history stretching back to the 18th century. In the period under study, this health insurer insured around 5 million people, of whom around 3 million (60\%) were female. This high percentage of female insurance holders may explain the almost balanced sex representation in our sample, even though the disease occurs much more commonly in men $[27,28]$.

The low rate of work incapacity certificates (7\%) may be attributable to the fact that we have older insurance holders who do not require a work incapacity certificate, as they may no longer be in gainful employment. Also, around $60 \%$ of the client base of the studied health insurer comprised women whose careers may have been put on hold to start a family. The inclusion of TB-related work incapacity certificates (e.g. due to bronchitis) has resulted in a substantial increase in the work incapacity certification rate to $19 \%$. Even so, this rate is still lower than would be expected given the age distribution. In addition to the high proportion of women, the low work incapacity certification rate may be due to the high proportion of unemployed insurance holders among the patients. Unemployment is also a risk factor for TB [6].

The DDDs were lower than recommended for the majority of patients. It is not possible based on the available data to determine whether these patients actually received fewer drugs or whether our method of calculating the DDDs resulted in an underestimation of the DDDs. The usage duration was not known for the last redeemed prescription and was not reflected in the calculation of the DDDs per day. This means that the drug treatment duration may be generally underestimated.

\section{Transferability}

With around 5 million insurance holders, the customers of DAK-Gesundheit represent just a fraction of the population covered by statutory health insurance in Germany. As a proportion of all statutory insurance holders in Germany, they accounted for a share of around 7\% during the study period. The historical development of DAK-Gesundheit is unusual and has produced a specific insurance clientele that cannot be considered to be representative of Germany. The reason of the lack in significance may stem from the heterogeneity of the population. So, for instance we could not prove an effect of sex on the amount of prescriptions. On the other hand, a high level of generalisation can be derived from the billing data, which are real valid prescription data. There is no doubt about the validity of the data and the conclusions drawn therefrom. While selection bias is to be expected, it can also be assumed that the effects found within the population can be also considered to be valid.

\section{Strengths and limitations}

The analyses allow a high level of generalisation that retains its validity beyond the specific study (high external validity). This is in contrast to clinical studies, which usually offer very low external validity. The study of the various diagnostic methods in terms of outpatient and inpatient diagnoses presents numerous opportunities for categorisation and different interpretations, and these in turn can lead to different results and conclusions. The most valid available information was used for this study. The disease definition resulted in the recognition of only confirmed diagnoses for outpatient diagnosis and main discharge diagnoses for inpatient diagnosis. The treatment of outpatients using TB-specific drugs has also been taken as an indication of $\mathrm{TB}$ infection, but does not allow for a study of the effect of the diagnosis. The methodological challenges included the differing lengths of data availability periods resulting from legal requirements. Outpatient diagnoses may only be stored in the health insurer's database for the index year minus 4 years, which in this context means that data were available for three claim years for our analyses. Hospital diagnoses are available for much longer, but the extra years cannot be placed into proportion with the corresponding outpatient diagnoses. For the hospital discharge diagnoses, the stay in hospital due to TB is known, but the actual supply of medication during the hospital stay is not documented in the claims data and is therefore a "black box" in analytical terms. There will almost certainly be differences in terms of how drug treatment is applied for TB in hospitals. A sputum examination with a positive 
laboratory result for TB generally results in drug treatment commencing sooner than sputum examinations with a negative result. However, from the fourth day, all patients can be assumed in practice to be on TB medication [16]. The method of assuming adequate medication only from the fourth day after diagnosis may well be a conservative estimate, but the data do not allow for differentiated modelling. Outpatient drug prescriptions were available for the entire 6-year study period for all patients included in the analysis.

The definition of affected patients takes a pragmatic approach that uses confirmed outpatient diagnoses, the administration of TB-specific drugs and a main diagnosis of pulmonary $\mathrm{TB}$ or miliary $\mathrm{TB}$ upon hospital discharge. The quality of ICD diagnoses for work incapacity certifications may not be sufficiently reliable for secondary data.

The compliance levels used for analysis can only be taken as an approximation of the doses actually taken as it is impossible to determine when and in which dose the drugs were actually taken.

There were inconsistent effects observed regarding prescription intensity (compliance) in the analysis. Our secondary statistical analyses indicated that prospective studies that took resistances to first-line drugs and comorbidity into particular account may be helpful. This is a field in which the factors of influence on the supply of drugs to TB patients in Germany have not yet been adequately described.

A direct review of compliance with guidelines (i.e. how consistent the actions of the prescribing physicians are with treatment guidelines) is not possible on the basis of claims data, but there was no indication of widespread contravention of the guidelines.

\section{Conclusions}

About $40 \%$ of all TB patients are diagnosed and treated in outpatient care, only. The doses of the prescribed four first-line drugs are higher for patients having received a main diagnosis of TB upon hospital discharge than for outpatients without previous hospitalisation. It should be analysed in further studies as to whether TB treatment for outpatients is sufficient regarding the doses of prescription first-line drugs.

Acknowledgement: The authors would like to thank DAK-Gesundheit for providing the database and for their valuable cooperation.

Role of data owners: A scientific employee of DAK-Gesundheit (SWR) organised the data transfer and offered suggestions on revising the draft manuscript. However, this did not result in any restrictions on how the data was presented.

Authors contributions: J.F. Kersten, A. Schablon, R. Diel and A. Nienhaus conceived the study. Data analysis was performed by J.F. Kersten and A. Schablon. The first draft of the paper was written by J.F. Kersten and A. Schablon. Important suggestions for the improvement of the first draft were provided by S. Wobbe-Ribinski, R. Diel and A. Nienhaus. All authors read and approved the manuscript.

Conflict of interest: J.F. Kersten has nothing to disclose. S. Wobbe-Ribinski has nothing to disclose. R. Diel reports personal fees from Bayer Vital outside the submitted work. A. Nienhaus has nothing to disclose. A. Schablon has nothing to disclose.

\section{References}

World Health Organization. Global Tuberculosis Report 2019. Geneva, World Health Organization, 2019.

2 Abel L, Fellay J, Haas DW, et al. Genetics of human susceptibility to active and latent tuberculosis: present knowledge and future perspectives. Lancet Infect Dis 2018; 18: e64-e75.

3 Della Bella C, Spinicci M, Grassi A, et al. Novel M. tuberculosis-specific IL-2 ELISpot assay discriminates adult patients with active or latent tuberculosis. Dieli F, editor. PLoS ONE 2018; 13: e0197825.

4 Cervantes J. Tuberculosis. Digging deep in the soul of humanity. Respir Med 2016; 119: 20-22.

5 Falzon D, Schünemann HJ, Harausz E, et al. World Health Organization treatment guidelines for drug-resistant tuberculosis, 2016 update. Eur Respir J 2017; 49: 1602308.

6 Schaberg T, Bauer T, Brinkmann F, et al. S2k-Leitlinie: Tuberkulose im Erwachsenenalter [Tuberculosis Guideline for Adults - Guideline for Diagnosis and Treatment of Tuberculosis including LTBI Testing and Treatment of the German Central Committee (DZK) and the German Respiratory Society (DGP)]. Pneumologie 2017; 71: 325-397.

7 Uplekar M, Raviglione M. WHO's end TB strategy: from stopping to ending the global TB epidemic. Indian J Tuberc 2015; 62: 196-199.

8 World Health Organization. The End TB Strategy. Geneva, World Health Organization, 2015

9 World Health Organization. Guidelines for Treatment of Drug-susceptible Tuberculosis and Patient Care, 2017 Update. Geneva, World Health Organization, 2017.

10 Feiterna-Sperling C, Brinkmann F, Adamczick C, et al. S2k-Leitlinie zur Diagnostik, Prävention und Therapie der Tuberkulose im Kindes- und Jugendalter [Consensus-Based Guidelines for Diagnosis, Prevention and Treatment of Tuberculosis in Children and Adolescents - A Guideline on Behalf of the German Society for Pediatric Infectious Diseases (DGPI)]. Pneumologie 2017; 71: 629-680.

11 Schaberg T, Bauer T, Castell S, et al. Empfehlungen zur Therapie, Chemoprävention und Chemoprophylaxe der Tuberkulose im Erwachsenen- und Kindesalter [Recommendations for therapy, chemoprevention and 
chemoprophylaxis of tuberculosis in adults and children. German Central Committee against Tuberculosis (DZK), German Respiratory Society (DGP)]. Pneumologie 2012; 66: 133-171.

12 Koenker R, Bassett G. Regression quantiles. Econometrica 1978; 46: 33.

13 Koenker RW. Confidence intervals for regression quantiles. In: Mandl P, Huskova M, eds. Asymptotic Statistics. New York, Springer-Verlag, 1994.

14 Koenker R. Quantile Regression. Cambridge, Cambridge University Press, 2005.

15 Huang D-Y, Hu Y-F, Wei N, et al. Innovative analysis of predictors for overall survival from systemic non-Hodgkin T cell lymphoma using quantile regression analysis. Chin Med J 2019; 132: 294-301.

16 Diel R, Nienhaus A, Hillemann D, et al. Cost-benefit analysis of Xpert MTB/RIF for tuberculosis suspects in German hospitals. Eur Respir J 2016; 47: 575-587.

17 Schubert I, Köster I, Küpper-Nybelen J, et al. Versorgungsforschung mit GKV-Routinedaten Bundesgesundheitsblatt Gesundheitsforsch Gesundheitsschutz 2008; 51: 1095-1105.

18 Bundesrepublik Deuschland. Zehntes Buch Sozialgesetzbuch. Germany. www.gesetze-im-internet.de/sgb_10/. Date last accessed: 12 July, 2020; date last updated: 12 July, 2020.

19 Ihle P, Köster I, Herholz H, et al. Versichertenstichprobe AOK Hessen/KV Hessen - Konzeption und Umsetzung einer personenbezogenen Datenbasis aus der Gesetzlichen Krankenversicherung. Das Gesundheitswes 2005; 67: 638-645.

20 Swart E, Thomas D, March S, et al. Erfahrungen mit der Datenverknüpfung von Primär- und Sekundärdaten in einer Interventionsstudie. Das Gesundheitswes 2011; 73: e126-e132.

21 Deutsches Institut für Medizinische Dokumentation und Information. ICD-10-GM Version 2020, status: 2020-3-23. Düsseldorf; 2020. www.dimdi.de/dynamic/de/klassifikationen/icd/icd-10-gm/ Date last accessed: 13 April 2020; date last updated: 23 March, 2020.

22 Das Fünfte Buch Sozialgesetzbuch - Gesetzliche Krankenversicherung, Dritter Abschnitt: Datenlöschung, Auskunftspflicht $\$ 304$ - (Artikel 1 des Gesetzes vom 20. Dezember 1988, BGBl. I S. 2477, 2482), das zuletzt durch Artikel 5 des Gesetzes vom 22. März 2020 (BGBl. I S. 604) geändert worden ist. www.gesetze-im-internet.de/sgb_ 5/SGB 5.pdf Date last accessed: April 13, 2020.

23 World Health Organization. Guidelines for ATC classification and DDD assignment 2019. Oslo, World Health Organization, 2018.

24 Fricke U, Günther J, Zawinell A, et al. Anatomisch-therapeutisch-chemische Klassifikation mit Tagesdosen für den deutschen Arzneimittelmarkt - Methodik der ATC-Klassifikation und DDD-Festlegung. Berlin, Wissenschaftliches Institut der AOK (WIdO), 2011

25 R Core Team. R: A Language and Environment for Statistical Computing. Vienna, R Foundation for Statistical Computing, 2019.

26 SAS Institute Inc. SAS. Version 9.4. Cary, SAS Institute Inc., 2014.

27 Robert Koch-Institut. Infektionsepidemiologisches Jahrbuch 2010. Berlin, 2011.

28 Mamelund S-E, Riise Bergsaker MA. Vaccine history, gender and influenza vaccination in a household context. Vaccine 2011; 29: 9441-9450. 